Check for updates

Cite this: RSC Adv., 2019, 9, 28870

\title{
Degradation of PVC waste into a flexible polymer by chemical modification using DINP moieties $\uparrow$
}

\author{
Lihui Lu, ${ }^{a}$ Shogo Kumagai, ${ }^{a}$ Tomohito Kameda, ${ }^{a}$ Ligang Luo (D) *b \\ and Toshiaki Yoshioka*a
}

In consideration of the toxicity and high migration capacity of plasticizers, the possibility to obtain flexible PVC via chemical modification of PVC was investigated for feedstock recycling. In this work, some $\mathrm{Cl}$ atoms of PVC were substituted with fragments of the common plasticizer DINP (diisononyl phthalate) in the presence of $\mathrm{K}_{2} \mathrm{CO}_{3}$ (potassium carbonate) or DIEA ( $N, N$-diisopropylethylamine), and the simultaneous elimination of PVC was suppressed. ${ }^{1} \mathrm{H}$ NMR $\left({ }^{1} \mathrm{H}\right.$ nuclear magnetic resonance spectroscopy) and ${ }^{1} \mathrm{H}-{ }^{1} \mathrm{H}$ COSY $\left({ }^{1} \mathrm{H}-{ }^{1} \mathrm{H}\right.$ correlation spectroscopy) were used to evaluate the substitution while a novel method of calculating the substitution and elimination ratios was developed using a combination of ${ }^{1} \mathrm{H}$ NMR and elemental analysis. A maximum substitution rate of $35.7 \%$ was achieved using thiophenol as a nucleophile in the presence of DIEA, while the corresponding elimination of $\mathrm{HCl}$ was just $4.4 \%$. In addition, the thermal stability of the modified PVCs was very close to that of pure PVC, which suggested that the main characteristics of PVC were preserved. Moreover, the $T_{g}$ values of all the modified PVCs were less than that of PVC, which means it is feasible to improve the plasticity of PVC via substituting some $\mathrm{Cl}$ on PVC with DINP moieties. Therefore, an alternative approach for feedstock recycling of PVC by chemical modification was developed in this work.

Received 4th July 2019

Accepted 27th August 2019

DOI: $10.1039 / \mathrm{c} 9 \mathrm{ra05081 \textrm {g }}$

rsc.li/rsc-advances chlorinated organic during pyrolysis treatment, which was limited various recycling demands. ${ }^{8}$ Therefore, a series of strategy were investigated on the migration of $\mathrm{Cl}$, such as thermal degradation, ${ }^{\mathbf{4}, \mathbf{9 1 1}}$ chemical modification, ${ }^{\mathbf{1 2 - 1 4}}$ hydrothermal process in supercritical or subcritical water, ${ }^{\mathbf{1 5 - 1 8}}$ nearcritical methanol process for dechlorination of PVC and additives recovery, ${ }^{19}$ and near-critical aqueous ammonia process. ${ }^{20}$ Among them, chemical modification of PVC is a famous technique that allows addition of new functionalities to PVC while maintaining its primary characteristics by substituting some $\mathrm{Cl}$ atoms with various nucleophilic reagents. ${ }^{21-24}$ In previous study, the properties of gas transport, ${ }^{21}$ luminescence, ${ }^{22}$ antibacterial, ${ }^{23}$ and plasticity ${ }^{24}$ have been improved by grafting different functional groups to substitute $\mathrm{Cl}$ atoms on PVC. In particular, P. Jia et al. investigated self-plasticization of PVC materials via chemical modification with phosphorus containing castor oil based derivatives, ${ }^{14}$ cardanol groups,${ }^{24}$ aminated tung oil methyl ester, ${ }^{25}$ and mannich base such as cardanol butyl ether ${ }^{13}$ and waste cooking oil methyl ester. ${ }^{26}$ These findings transformed the traditional plastic processing technology to obtain cleaner production of internally plasticized PVCs. Therefore, this technique has a great potential to be used for PVC recycling because it can convert waste PVC into value-added materials with improved properties, which can be referred to "upgrade recycling".

Without plasticizer, $\mathrm{PVC}$ is a rigid polymer at room temperature. Nevertheless, PVC products are normally obtained

$\dagger$ Electronic supplementary information (ESI) available. See DOI: 10.1039/c9ra05081g 
from the combination of PVC with additives which provides our desired properties to the material. ${ }^{27}$ The plasticizers, which act as spacer between molecules of the polymer, are mainly used to reduce the melt viscosity, to decrease the transition temperature or to lower the elastic modulus of PVC as it weakens the intermolecular bonds of the polymer which facilitate its processing. ${ }^{27}$ The most commonly used plasticizer were terephthalate species, such as diisononyl phthalate (DINP), diethylhexyl phthalate (DEHP), diisobutyl phthalate (DIBP), di- $n$-butyl phthalate (DBP), and diisodecyl phthalate (DIDP). ${ }^{28}$

However, most of these plasticizers have been banned in the last decades due to their high migration capacity and strong toxicity. ${ }^{29,30}$ Therefore, the possibility to produce flexible PVC by chemical modification using common plasticizer DINP moieties as nucleophiles (Fig. 1), which was investigated in this work for feedstock recycling. The obtained products are environmentallyfriendly while compared with a mixture of PVC and plasticizer. However, the main problem is the elimination reaction simultaneously occurring with a strong base such like sodium hydroxide $(\mathrm{NaOH}),{ }^{31}$ which have effect on the properties of PVC and causes a blackish color, which will narrow the applicability of the recycled PVC. As a result, the effect of potassium carbonate $\left(\mathrm{K}_{2} \mathrm{CO}_{3}\right)$ and $N, N$-diisopropylethylamine (DIEA) were investigated as a weak inorganic base and organic base on substitution and elimination, respectively. The substitution was evaluated by ${ }^{1} \mathrm{H}$ nuclear magnetic resonance $\left({ }^{1} \mathrm{H}\right.$ NMR). Moreover, a novel method of calculating the substitution and elimination rates based on ${ }^{1} \mathrm{H}$ NMR and elemental analysis was developed, which is simpler and more accurate than the methods used in previous study. ${ }^{32}$

\section{Results and discussion}

\section{Confirmation of the substitution of PVC with thiophenol}

At first, the FT-IR spectra were employed to investigate the structure between PVC and the modified PVC with thiophenol (Fig. S1†). The sharp peaks of PVC at 2969, 2920, 1436 and $606 \mathrm{~cm}^{-1}$ are belong to the alkane $\mathrm{C}-\mathrm{H}$ stretching vibration, $-\mathrm{CH}_{2}$ deformation vibration and $\mathrm{C}-\mathrm{Cl}$ stretching vibration, respectively. ${ }^{13,33}$ Compared to the curve of PVC, it was observed that several new peaks at 3075,1575 and $1552 \mathrm{~cm}^{-1}$ were appeared, which are attributed to $\mathrm{C}-\mathrm{H}$ stretching vibration of arene and the skeleton vibration of benzene ring, respectively. ${ }^{34}$

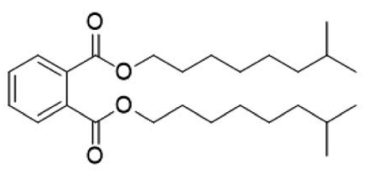

DINP (Diisononyl Phthalate)

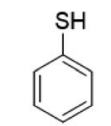

Thiophenol

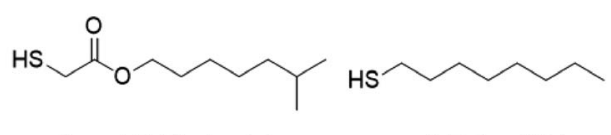

Isooctyl Thioglycolate

1-Octanethiol
Fig. 1 Structures of DINP and the nucleophiles investigated in this work.
This was suggested that the thiophenol group was successfully grafted on PVC backbone, which means the nucleophilic substitution of PVC and thiophenol was efficient in the presence of $\mathrm{K}_{2} \mathrm{CO}_{3}$ as a base.

Subsequently, the ${ }^{1} \mathrm{H}$ NMR spectra was used to identify the PVC and the modified PVC with thiophenol. In Fig. 2, one of common signal of the chemical shift between $2.25-2.52 \mathrm{ppm}$ is attributed to hydrogen atoms a, and the other common signal of the chemical shift between $4.35-4.52 \mathrm{ppm}$ is attributed to hydrogen atom b. ${ }^{22}$ Compared to the spectrum of PVC (Fig. 2(A)), there were new peaks of hydrogen atoms $\mathrm{c}$ and $\mathrm{e}$ observed in Fig. 2(B). It is obviously noted that a single sharp peak of the chemical shift appeared around $3.66 \mathrm{ppm}$, which is attributed to the hydrogen atom $\mathrm{c}$ of the new produced group (-CH-S-) of the modified PVC with thiophenol. It was suggested that a portion of chlorine atoms were already substituted by thiophenol and the nucleophilic substitution occurred. Moreover, the signals of hydrogen atoms e are also observed at the chemical shifts between 7.25-7.50 ppm.

On the other hand, the peak of hydrogen atoms $\mathrm{d}(\mathrm{CH}=\mathrm{CH})$ is not obviously observed in Fig. 2(B), which was suggested that the simultaneous elimination of PVC was suppressed under the reaction condition. Therefore, the rate of dechlorination was consistent with the ratios calculated from elemental analysis, which was according to the previously reported methods, ${ }^{32}$ and then the corresponding elimination rate could be obtained. Nevertheless, substitution ratio was calculated from their ${ }^{1} \mathrm{H}$ NMR spectra. In Fig. 2(B), it was showed that the chemical shifts between 7.25-7.50 ppm is attributed to the hydrogen atoms e (five hydrogen atoms) which are from thiophenol before the reaction, while the chemical shifts between $1.95-2.35 \mathrm{ppm}$ is attributed to the hydrogen atoms a (two hydrogen atoms) which are from PVC before the reaction. Therefore, the substitution ratio was defined as eqn (1):

$$
\text { Substitution ratio }[\%]=(x / m) /(y / n) \times 100 \%
$$

where $x$ and $y$ are the integrals of the corresponding hydrogen atoms shown in Fig. 2(B), and $m=5$ and $n=2$ when PVC was

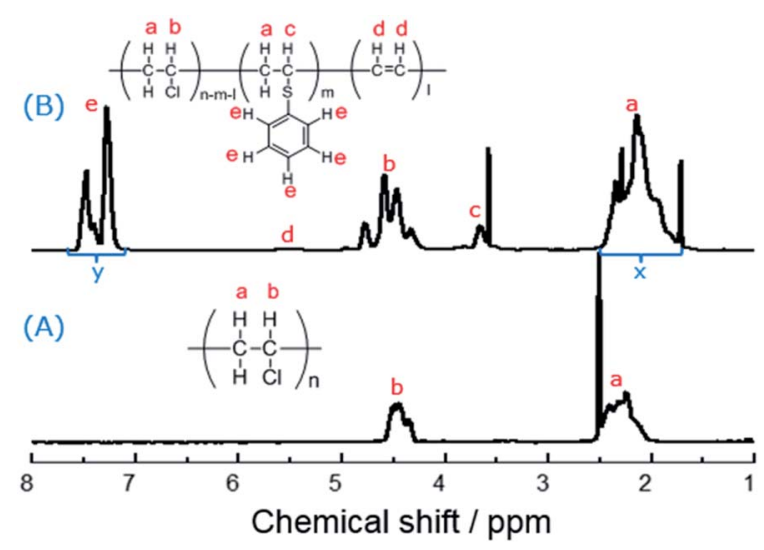

Fig. $2 \quad{ }^{1} \mathrm{H}$ NMR spectra of PVC and the modified PVC using thiophenol as a nucleophile $\left(400 \mathrm{MHz}, \mathrm{THF}-\mathrm{d}_{8}, 329.9 \mathrm{~K}\right.$. Reaction conditions: $500 \mathrm{mg}$ PVC, 1.0 eq. thiophenol, 0.1 eq. $\mathrm{K}_{2} \mathrm{CO}_{3}, 40{ }^{\circ} \mathrm{C}, 50 \mathrm{~mL}$ DMF as solvent, $3 \mathrm{~h}$ ). 
substituted with thiophenol. The elimination ratio was determined by subtracting the substitution ratio from the dechlorination ratio, as shown in eqn (2):

Elimination ratio $[\%]=$ dechlorination ratio $[\%]-$ substitution ratio $[\%]$

To further confirm the substitution of PVC with thiophenol, ${ }^{1} \mathrm{H}-{ }^{1} \mathrm{H}$ COSY was also performed for characterization of the products. In Fig. 3, the correlation peaks (marked with red circles) between hydrogen atoms a and c are observed obviously. It was indicated that hydrogen atoms a and $\mathrm{c}$ were correlated each other and hydrogen atom $\mathrm{c}$ was bonding with the adjacent carbon of hydrogen atoms a, which was demonstrated that the structure of $\mathrm{C}_{6} \mathrm{H}_{5}-\mathrm{S}-\mathrm{CH}-\mathrm{CH}_{2}-$ was successfully formed. According to the above results, it was considered that thiophenol was attacked to the $\alpha$-position carbon of PVC to replace $\mathrm{Cl}$ atom and produce $\mathrm{HCl}$, which is corresponding with the previous literature. ${ }^{9,12}$ Nevertheless, the produced $\mathrm{HCl}$ could be neutralized by the base of $\mathrm{K}_{2} \mathrm{CO}_{3}$ or DIEA. Therefore, one part of $\mathrm{Cl}$ atoms in PVC were successfully substituted by thiophenol, which is in accordance with the experimental result from ${ }^{1} \mathrm{H}$ NMR spectrum in Fig. 2(B) and Scheme 1.

\section{Characterization of the modified PVCs}

Subsequently, isooctyl thioglycolate and 1-octanethiol were conducted as nucleophilic reagents to modify the PVC sample. And the dechlorination degrees of PVC using the three different nucleophiles in DMF using $\mathrm{K}_{2} \mathrm{CO}_{3}$ or DIEA as bases were summarized in Table 1. When taking thiophenol as a nucleophile, the rate of dechlorination was $20.2 \%$ in the presence of $\mathrm{K}_{2} \mathrm{CO}_{3}$ (Table 1, entry 1 ), while the dechlorination rate was $40.1 \%$ with DIEA as a base (Table 1, entry 2). This was attributed to the corresponding high substitution ratios of $17.5 \%$ and $35.7 \%$, respectively. However, when using isooctyl thioglycolate and 1octanethiol in the presence of $\mathrm{K}_{2} \mathrm{CO}_{3}$, the dechlorination ratios were $7.0 \%$ (Table 1 , entry 3 ) and $10.7 \%$ (Table 1 , entry 5 ),

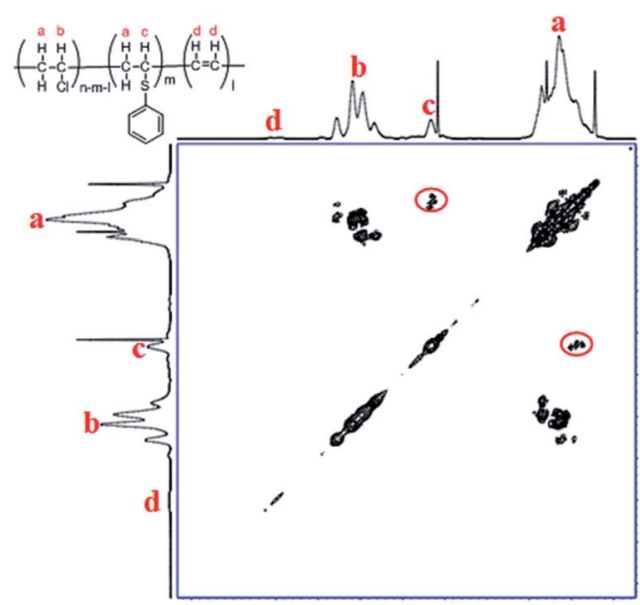

Fig. $3{ }^{1} \mathrm{H}-{ }^{1} \mathrm{H}$ COSY of PVC and the modified PVC with thiophenol (400 MHz, THF- $d_{8}, 353.0 \mathrm{~K}$. Reaction conditions: $500 \mathrm{mg}$ PVC, 1.0 eq. thiophenol, $0.1 \mathrm{eq} . \mathrm{K}_{2} \mathrm{CO}_{3}, 40{ }^{\circ} \mathrm{C}, 50 \mathrm{~mL} \mathrm{DMF}$ as solvent, $3 \mathrm{~h}$ ).

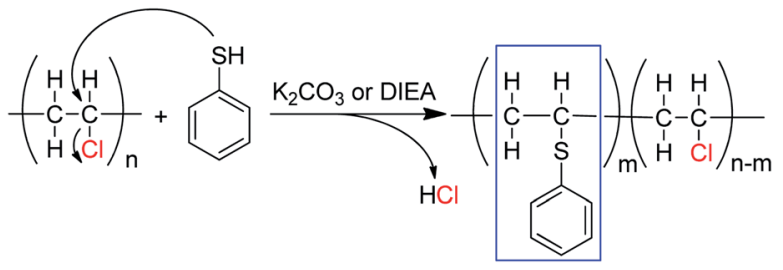

Scheme 1 Substitution reaction of PVC with thiophenol in the presence of $\mathrm{K}_{2} \mathrm{CO}_{3}$ or DIEA.

respectively. This was because their corresponding substitution rates were just $6.5 \%$ and $6.3 \%$, respectively. While using DIEA as a base, the degree of substitution for isooctyl thioglycolate was just 3.7\% (Table 1, entry 4), and there was no reaction using 1octanethiol (Table 1, entry 6), which was showed lower efficiency. Therefore, the efficiency condition of substitution was using thiophenol as the nucleophile because the nucleophilic group $\mathrm{C}_{6} \mathrm{H}_{5} \mathrm{~S}$ - is more easily formed under the reaction condition due to the conjugation of the benzene ring. In addition, it was suggested that the effect of isooctyl thioglycolate was shown better than that of 1-octanethiol as the nucleophile when both organic and inorganic bases were used, probably due to the positive influence of electron-withdrawing carbonyl group $(\mathrm{C}=\mathrm{O})$. Furthermore, when thiophenol was the nucleophile, a better performance was obtained using the organic base DIEA, whereas the inorganic base $\mathrm{K}_{2} \mathrm{CO}_{3}$ was a better base when isooctyl thioglycolate and 1octanethiol were used, as shown in Table 1. Finally, all the elimination ratios of the modified PVC were lower than their corresponding substitution ratios, probably due to the relatively weak basicity of $\mathrm{K}_{2} \mathrm{CO}_{3}$ and DIEA in comparison with $\mathrm{NaOH}^{17}$

\section{Thermal stability of PVC and the modified PVCs}

For confirmation of the thermal stabilities of pure PVC and the modified PVC with different nucleophilic reagents using DIEA as a base, the thermo-gravimetric and differential thermal analysis (TG-DTA) of PVC sample were conducted in Fig. 4. At first, it was observed that the PVC used in these experiments showed a weight loss of $5 \mathrm{wt} \%$ at an onset temperature ( $\left.T_{\mathrm{D} \text {-onset }}\right)$ of $269^{\circ} \mathrm{C}$. This may be led by dehydrochlorination of PVC in the temperature range of about $250-350{ }^{\circ} \mathrm{C}$ in the main reaction of the first stage, which is corresponding with the literature. ${ }^{4}$ However, a weight loss of $63.1 \mathrm{wt} \%$ was observed for unmodified PVC after the first degradation stage, suggesting that the formed polyene chain could also decomposed during the first stage. This is because that $c a$. 57 wt $\%$ of weight loss was expected from the complete elimination of PVC. As shown in Fig. 4, it was indicated that the thermal degradation of the modified PVCs with different nucleophilic groups and dechlorination ratios were showed the similar trend as that of pure PVC, which also include two distinct stages. Moreover, the product modified by thiophenol with $40.1 \%$ dechlorination ratio showed a similar degradation character to unmodified PVC during the first stage. And its $T_{\mathrm{D}-o n s e t}$ is $261{ }^{\circ} \mathrm{C}$ and the weight loss of the first stage is $63.1 \%$. However, it showed a different degradation behavior during the second degradation 
Table 1 Degrees of dechlorination, substitution and elimination for the chemical modification of PVC using different nucleophiles and bases ${ }^{a}$

\begin{tabular}{|c|c|c|c|c|c|}
\hline Entry & Nucleophile & Base & Dechlorination $^{b}(\%$, EA) & Substitution $^{c}\left(\%,{ }^{1} \mathrm{H}\right.$ NMR $)$ & $\begin{array}{l}\text { Elimination } \\
(\%)\end{array}$ \\
\hline 1 & Thiophenol & $\mathrm{K}_{2} \mathrm{CO}_{3}{ }^{d}$ & 20.2 & 17.5 & 2.7 \\
\hline 3 & \multirow[t]{2}{*}{ Isooctyl thioglycolate } & $\mathrm{K}_{2} \mathrm{CO}_{3}{ }^{d}$ & 7.0 & 6.5 & 0.5 \\
\hline 4 & & DIEA $^{e}$ & 5.1 & 3.7 & 1.4 \\
\hline 5 & 1-Octanethiol & $\mathrm{K}_{2} \mathrm{CO}_{3}{ }^{d}$ & 10.7 & 6.3 & 4.4 \\
\hline
\end{tabular}

${ }^{a}$ Reaction conditions: $500 \mathrm{mg}$ PVC, 1.0 eq. appropriate nucleophiles, 0.1 eq. $\mathrm{K}_{2} \mathrm{CO}_{3}$ or 1.0 eq. DIEA, $40{ }^{\circ} \mathrm{C}, 50 \mathrm{~mL}$ DMF as solvent, $3 \mathrm{~h} .{ }^{b}$ Results were from elemental analysis. ${ }^{c}$ Results were from ${ }^{1} \mathrm{H}$ NMR analysis. ${ }^{d}$ Reaction temperature: $40{ }^{\circ} \mathrm{C}$. ${ }^{e}$ Reaction temperature: $110{ }^{\circ} \mathrm{C}$.

stage, the temperature range of PVC modified by thiophenol with $40.1 \%$ was around $350-480{ }^{\circ} \mathrm{C}$, while the temperature range of the second stage on PVC was approximate $350-525{ }^{\circ} \mathrm{C}$, which was corresponding with the cracking and decomposition of the dehydrochlorinated PVC. ${ }^{4}$ The possible cause might be the high substitution ratio $(35.7 \%)$ and the maximum rate of dechlorination $(40.1 \%)$ resulted from it. Simultaneously, the thermal stability of the products modified by isooctyl thioglycolate and 1-octanethiol with the corresponding dechlorination ratio of $5.1 \%$ and $1.0 \%$ were slightly reduced, resulting in the $T_{\mathrm{D} \text {-onset }}$ of $241{ }^{\circ} \mathrm{C}$ and $236{ }^{\circ} \mathrm{C}$, respectively. It is worth noting that their degradation behaviors remained identical with that of pure PVC during the second stage, probably due to their low dechlorination concentration.

To further investigate the effect of the substituted concentration on thermal stability, the PVC substituted with thiophenol and 1-octanethiol were also conducted by TGA analysis. As shown in Fig. 5, it showed that the same degradation character was observed during the first stage $\left(250-350{ }^{\circ} \mathrm{C}\right)$ for both thiophenol-substituted PVCs, even with $17.5 \%$ and $35.7 \%$ substitution rates, respectively. Nevertheless, the trends for those degradation had a significantly difference during the second stage $\left(350-480{ }^{\circ} \mathrm{C}\right)$, which the sample with $17.5 \%$ substitution has loss over $20 \mathrm{wt} \%$ weight. It was because that

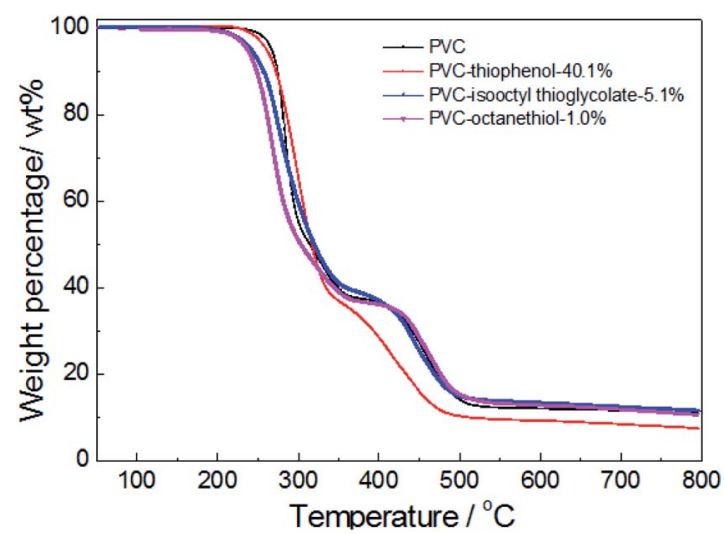

Fig. 4 TG analysis of PVC and the modified PVCs with different nucleophiles using DIEA as a base (testing conditions: sample, $10 \mathrm{mg}$; heating temperature, $50-800{ }^{\circ} \mathrm{C}$; heating rate, $10{ }^{\circ} \mathrm{C} \min ^{-1} ; 50$ $\mathrm{mL} \min ^{-1} \mathrm{~N}_{2}$ flow). the sample with $17.5 \%$ substitution ratio did not have a PVClike character, which might be attributed to the effect of the different substituted rates or aromatic nucleophilic group $\left(\mathrm{C}_{6} \mathrm{H}_{5} \mathrm{~S}-\right)$ itself. Then, the sample with $17.5 \%$ substitution also has a significant degradation in the third stage.

In Fig. 6, the thermal stability of both 1-octanethiolsubstituted PVCs slightly reduced in comparison with that of PVC during the first stage, resulting in their $T_{\mathrm{D}-\text {-onset }}$ of $236^{\circ} \mathrm{C}$ and $235{ }^{\circ} \mathrm{C}$. However, the product with no substitution rate showed the similar trend as PVC during the second stage, while the modified PVC product with $6.3 \%$ substitution rate was showed easily degradable. It was implied that the thermal stability was slightly decreased on PVC modified with grafting an aliphatic nucleophilic group.

\section{Glass transition temperature ( $\left.T_{\mathrm{g}}\right)$ of PVC and the modified PVCs}

In addition, the glass transition temperature $\left(T_{\mathrm{g}}\right)$ of PVC and modified PVCs were also detected using DSC measurements, and the results were summarized in Table 2. It was observed that the $T_{\mathrm{g}}$ of pure PVC with a stiff backbone is at around $82{ }^{\circ} \mathrm{C}$ (Table 2, entry 1). The addition of plasticizer into PVC polymer will increase the distance of PVC chains and make the

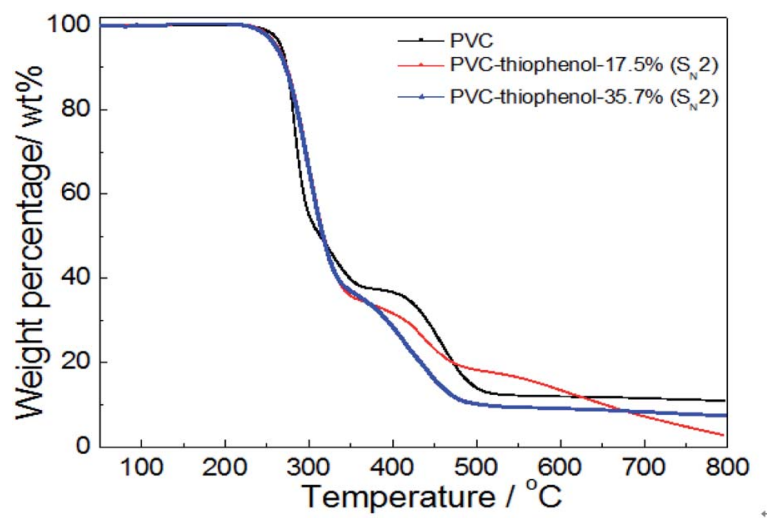

Fig. 5 TG analysis of PVC and the modified PVCs with thiophenol using $\mathrm{K}_{2} \mathrm{CO}_{3}$ or DIEA as a base (testing conditions: sample, $10 \mathrm{mg}$; heating temperature, $50-800{ }^{\circ} \mathrm{C}$; heating rate, $10{ }^{\circ} \mathrm{C} \mathrm{min}^{-1} ; 50$ $\mathrm{mL} \min ^{-1} \mathrm{~N}_{2}$ flow). 


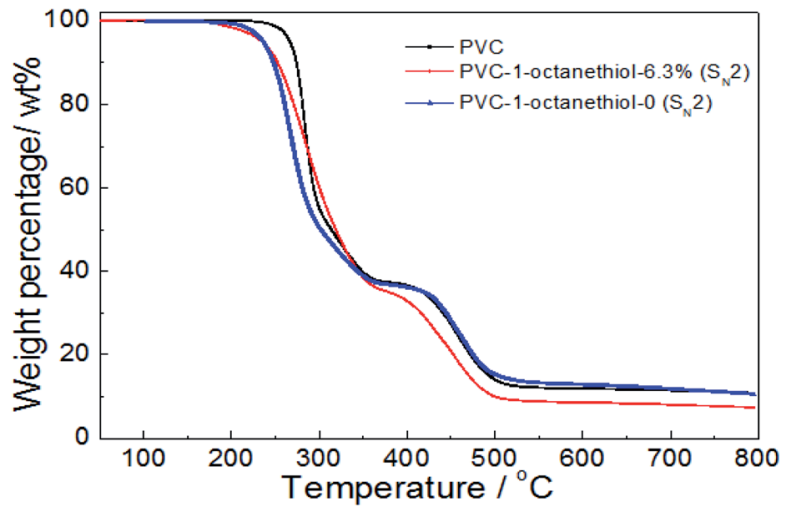

Fig. 6 TG analysis of PVC and the modified PVCs with 1-octanethiol using $\mathrm{K}_{2} \mathrm{CO}_{3}$ or DIEA as a base (testing conditions: sample, $10 \mathrm{mg}$; heating temperature, $50-800{ }^{\circ} \mathrm{C}$; heating rate, $10{ }^{\circ} \mathrm{C} \mathrm{min}^{-1} ; 50$ $\mathrm{mL} \min ^{-1} \mathrm{~N}_{2}$ flow).

macromolecular structure polymer easy to move, increase the free volume of PVC and reduce the $T_{\mathrm{g}}{ }^{13}$ Obviously, the $T_{\mathrm{g}}$ of all the modified PVCs are less than that of pure PVC, as shown in Table 2. This indicates that internally plasticized PVCs can be obtained via chemical modification of PVC with appropriate nucleophilic reagents (DINP moieties). In addition, the $T_{\mathrm{g}}$ of the modified PVCs with thiophenol slightly decreased compared to pure PVC. Nevertheless, the modified PVC with $35.7 \%$ substitution ratio has a lower $T_{\mathrm{g}}$ (Table 2, entry 3 ) in comparison with the one with $17.5 \%$ substitution ratio (Table 2, entry 2), which suggests high substitution efficiency can be conducive to improving plasticity of PVC. Moreover, the $T_{\mathrm{g}}$ of the modified PVCs with long carbon chain nucleophiles (isooctyl thioglycolate and 1-octanethiol) distinctly decrease to 65.8 and $65.6{ }^{\circ} \mathrm{C}$ (Table 2, entries 4 and 6), respectively. It means that the effect of long carbon chain group on improving the plasticity of PVC are evidently better than that of thiophenol group. Therefore, these results illustrate that the nucleophiles of DINP moieties can play an internally plasticized effect on PVC through replacing partial $\mathrm{Cl}$ atoms on PVC. Besides, the factor for decreasing $T_{\mathrm{g}}$ is mainly caused that the substituting of $\mathrm{Cl}$ atoms with nucleophiles will increase distance between PVC chains and reduce intermolecular force. ${ }^{24-26}$

Table 2 DSC and TGA data of PVC and modified PVCs

\begin{tabular}{llll}
\hline Entry & Samples $\left(\mathrm{S}_{\mathrm{N}_{2}}\right)$ & $T_{\mathrm{g}}{ }^{a}\left({ }^{\circ} \mathrm{C}\right)$ & $T_{\mathrm{D}}{ }^{b}\left({ }^{\circ} \mathrm{C}\right)$ \\
\hline 1 & PVC & 82.1 & 269 \\
2 & PVC-thiophenol-17.5\% & 77.7 & 263 \\
3 & PVC-thiophenol-35.7\% & 75.2 & 261 \\
4 & PVC-isooctyl thioglycolate-6.5\% & 65.8 & 265 \\
5 & PVC-isooctyl thioglycolate-3.7\% & 75.6 & 241 \\
6 & PVC-1-octanethiol-6.3\% & 65.6 & 236
\end{tabular}

${ }^{a}$ DSC testing conditions: each sample, $10 \mathrm{mg}$; heating temperature, -50 to $+120{ }^{\circ} \mathrm{C}$; heating rate, $10{ }^{\circ} \mathrm{C} \mathrm{min}^{-1}$; under $\mathrm{N}_{2}$ atmosphere. ${ }^{b}$ TGA testing conditions: sample, $10 \mathrm{mg}$; heating temperature, 50-

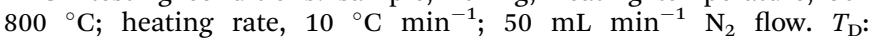
temperature of weight loss of $5 \mathrm{wt} \%$ for a sample.

\section{Experimental}

\section{Materials}

PVC powder $\left(M_{\mathrm{w}}=68750\right), \mathrm{K}_{2} \mathrm{CO}_{3}$, DIEA, thiophenol, isooctyl thioglycolate and dimethylformamide (DMF) were purchased from the Wako Pure Chemical Industries, Ltd (Japan). Tetrahydrofuran (THF), 1-octanethiol and methanol were acquired from the Kanto Chemical Co., Inc. (Japan). Thiophenol, isooctyl thioglycolate, and 1-octanethiol were used as nucleophilic reagents. Water was used the ultrapure water in the experiments. All other reagents and solvents were used without further purification.

\section{Experimental procedure}

In a type experiment, PVC powder $(500 \mathrm{mg}, 8 \mathrm{mmol}$ equivalent monomer), an appropriate nucleophile (1.0 eq.), and a base (either $\mathrm{K}_{2} \mathrm{CO}_{3}$ (0.1 eq.) or DIEA (1.0 eq.)) were added to $50 \mathrm{~mL}$ of DMF in a three-neck flask while stirring. The reaction mixture was heated respectively up to $40{ }^{\circ} \mathrm{C}$ or $110{ }^{\circ} \mathrm{C}$ using a silicone oil bath for $3 \mathrm{~h}$. After reaching the required reaction time, the solution was filtered and washed with methanol to obtain a precipitate. The precipitate was filtered off and dissolved in THF to remove impurities. This was poured into methanol for recrystallization, after which the white solid was filtered off and dried under reduced pressure to further analyze. Each experiment was repeated at least 2 times (in duplicate) unless otherwise noted.

\section{Product analysis}

The purified products were analyzed by ${ }^{1} \mathrm{H}$ NMR (Bruker DPX400, $400 \mathrm{MHz}$ ), and deuterated solvents were THF- $\mathrm{d}_{8}$ and $\mathrm{DMF}_{-} \mathrm{d}_{7}$. In addition, elemental analysis of the products was performed using a CHN-coder MT6 (Japan Yanaco New Science Inc.). Fourier transform infrared spectrometry (FT-IR) was recorded on a NICOLET iS10 Fourier transformed infrared spectrophotometer. The thermal stability of the products (10 $\mathrm{mg}$ ) was measured using a TG/DTA 6200 (Japan Seiko Instruments) by heating from $50-800{ }^{\circ} \mathrm{C}$ at a heating rate of $10{ }^{\circ} \mathrm{C} \min ^{-1}$ under $\mathrm{N}_{2}$ flow $\left(50 \mathrm{~mL} \min ^{-1}\right)$. Glass transition temperature $\left(T_{\mathrm{g}}\right)$ was characterized using a NETZSCH DSC 200 PC analyser (differential scanning calorimeter measurements) under $\mathrm{N}_{2}$ atmosphere. The temperature was over a range of -50 to $+120{ }^{\circ} \mathrm{C}$ with a heating of $10{ }^{\circ} \mathrm{C} \mathrm{min}{ }^{-1}$. Approximately $10 \mathrm{mg}$ of PVC materials were weighed and sealed in a $40 \mu \mathrm{L}$ aluminum crucible and immediately detected using DSC measurement. The DSC data was collected from a first cycle of heating. ${ }^{25,26}$

\section{Conclusions}

In this paper, thiophenol or long carbon chain groups in the presence of an inorganic base $\left(\mathrm{K}_{2} \mathrm{CO}_{3}\right)$ and an organic base (DIEA) were successfully employed to remove the $\mathrm{Cl}$ in PVC, which convert waste PVC into value-added products to improve its plasticity for feedstock recycling. The maximum substitution ratio reached up to $35.7 \%$ when using thiophenol in the presence of DIEA, which was probably attributed to the easily 
formed nucleophilic group $\left(\mathrm{C}_{6} \mathrm{H}_{5} \mathrm{~S}-\right)$. Meanwhile, the elimination ratio was just $4.4 \%$ due to the use of a weak base. It was observed that there was a slight change in the thermal stability of all the modified PVC during the first degradation stage when compared to pure PVC, which suggested that the main chemical property of PVC remained after chemical modification. Moreover, it is feasible to improve plasticity of PVC via replacing some $\mathrm{Cl}$ atoms by DINP moieties reagents because the $T_{\mathrm{g}}$ of all the modified PVCs in this work were less than that of PVC.

\section{Conflicts of interest}

There are no conflicts to declare.

\section{Acknowledgements}

This work was supported by the Natural Science Foundation of China (Grant No. 31801321) and sponsored by Shanghai Sailing Program (Grant No. 19YF1436300).

\section{Notes and references}

1 R. C. Thompson, C. J. Moore, F. S. Saal and S. H. Swan, Philos. Trans. R. Soc., B, 2009, 364, 2153; A. H. Tullo, Chem. Eng. News, 2015, 96, 16; J. R. Jambeck, G. Roland, W. Chris, R. S. Theodore, P. Miriam, A. Anthony, N. Ramani and L. Kara, Science, 2015, 347, 768.

2 R. Geyer, J. R. Jambeck and K. L. Law, Sci. Adv., 2017, 3, e1700782; M. Bläsing and W. Amelung, Sci. Total Environ., 2018, 15, 422; J. Aguado, D. P. Serrano and J. M. Escala, Ind. Eng. Chem. Res., 2008, 47, 7982-7992; J. Lu, S. Ma and J. Gao, Energy Fuels, 2002, 16, 1251.

3 J. Yu, L. Sun, C. Ma, Y. Qiao and H. Yao, Waste Manag., 2016, 48, 300; N. R. Janjua, G. K. Mortensen, A. M. Andersson, B. Kongshoj, N. E. Skakkebak and H. C. Wulf, Environ. Sci. Technol., 2007, 41, 5564.

4 J. Chen, Z. Liu, J. Jiang, X. Nie and Y. Zhou, RSC Adv., 2015, 5, 56171; J. Yu, L. Sun, C. Ma, Y. Qiao and H. Yao, Waste Manag., 2016, 48, 300 .

5 P. Tiemblo, J. Guzman, E. Riande, C. Mijangos and H. Reinecke, Polymer, 2001, 42, 4817.

6 P. Tiemblo, J. Guzman, E. Riande, C. Mijangos and H. Reinecke, Macromolecules, 2002, 35, 420.

7 N. Garcia, M. Hoyos, G. Teyssedre, R. Navarro, H. Reinecke and P. Tiemblo, Polym. Degrad. Stab., 2007, 92, 2300.

8 T. Kameda, M. Ono, G. Grause, T. Mizoguchi and T. Yoshioka, J. Polym. Res., 2011, 18, 945; K. W. Lee, J. W. Chung and S. Kwak, Macromol. Rapid Commun., 2016, 37, 2045.

9 X. Zhang, L. Tang, N. Zhang, Q. Gao, C. Zhang and Z. Zhu, Energy Fuels, 2003, 17, 896.
10 M. Sadat-Shojai and G.-R. Bakhshandeh, Polym. Degrad. Stab., 2011, 96, 404.

11 B. Gui, Y. Qiao, D. Wan, S. Liu, Z. Han, H. Yao and M. Xu, Proc. Combust. Inst., 2013, 34, 2321.

12 P. H. Daniels, J. Vinyl Addit. Technol., 2009, 15, 219.

13 P. Jia, L. Hu, Q. Shang, R. Wang, M. Zhang and Y. Zhou, ACS Sustainable Chem. Eng., 2017, 5, 6665.

14 P. Jia, L. Hu, M. Zhang, G. Feng and Y. Zhou, Eur. Polym. J., 2017, 87, 209.

15 Y. Qi, J. He, F. Xiu, W. Nie and M. Chen, J. Cleaner Prod., 2018, 196, 331.

16 Z. Yao and X. Ma, Bioresour. Technol., 2018, 247, 504.

17 P. Zhao, Z. Li, T. Li, W. Yan and S. Ge, J. Cleaner Prod., 2017, $152,38$.

18 N. Huang, P. Zhao, S. Ghosh and A. Fedyukhin, Appl. Energy, 2019, 240, 882.

19 Y. Qi, J. He, Y. Li, X. Yu, F. Xiu, Y. Deng and X. Gao, Waste Manag., 2018, 80, 1.

20 F. Xiu, Y. Li, Y. Qi, X. Yu, J. He, Y. Lu, X. Gao, Y. Deng and Z. Song, Waste Manag., 2019, 84, 355.

21 P. Tiemblo, J. Guzman, E. Riande, C. Mijangos and H. Reinecke, Macromolecules, 2002, 35, 420.

22 N. Garcia, M. Hoyos, G. Teyssedre, R. Navarro, H. Reinecke and P. Tiemblo, Polym. Degrad. Stab., 2007, 92, 2300.

23 T. Kameda, M. Ono, G. Grause, T. Mizoguchi and T. Yoshioka, J. Polym. Res., 2011, 18, 945.

24 P. Jia, M. Zhang, L. Hu, R. Wang, C. Sun and Y. Zhou, Polymers, 2017, 9, 621.

25 P. Jia, L. Hu, X. Yang, M. Zhang, Q. Shang and Y. Zhou, RSC Adv., 2017, 7, 30101.

26 P. Jia, M. Zhang, L. Hu, F. Song, G. Feng and Y. Zhou, Sci. Rep., 2018, 8, 1589.

27 N. V. Gama, R. Santos, B. Godinho, R. Silva and A. Ferreira, J. Polym. Environ., 2019, 27, 703; B. L. Shah and V. V. Shertukde, J. Appl. Polym. Sci., 2003, 90, 3278.

28 J. Borch, M. Axelstad, A. M. Vinggaard and M. Dalgaard, Toxicol. Lett., 2006, 163, 183.

29 . Y. Suarez, R. P. C. Narváez and J. P. Corriou, J. Ind. Eng. Chem., 2014, 20, 198.

30 K. Jaakkola and T. L. Knight, Environ. Health Perspect., 2008, 116, 845 .

31 T. Yoshioka, T. Kameda, I. Shogo and O. Akitsugu, Polym. Degrad. Stab., 2008, 93, 1138.

32 M. Yoshihara, G. Grause, T. Kameda and T. Yoshioka, J. Mater. Cycles Waste Manage., 2010, 12, 264.

33 T. Kameda, M. Ono, G. Grause, T. Mizoguch and T. Yoshioka, Polym. Degrad. Stab., 2009, 94, 107.

34 E. Pretsch, P. Buhlmann and C. Affolter, Structure Determination of Organic Compounds: Table of Spectral Data, Springer-Verlag, Berlin Heidelberg, 2000, p. 271. 